\title{
UNCERTAINTIES OF THE ESTIMATORS AND PARAMETERS OF DISTRIBUTION IN MEASUREMENTS WITH MULTIPLY OBSERVATIONS
}

\author{
Mykhaylo Dorozhovets, Dr.Sc., Prof., e-mail: michdor@prz.edu.pl; Anna Szlachta, Ph.D., Ass.-Prof., \\ Rzeszow University of Technology, Poland
}

\begin{abstract}
The article shows that the commonly used method of estimating the Type A uncertainty of measurements based on the standard deviation of estimators of population parameters does not meet the definition of uncertainty. For correct determination of the standard uncertainty, it is necessary to use the distribution of the corresponding population parameter at the values of population estimators determined from the experiment but not the probability distribution of the estimator. The joint probability distribution of population parameters can be derived by transforming the joint distribution of estimators using a Jacobian equal to the ratio of the scale parameter estimator to the population scale parameter itself. Independently on population distribution, the standard uncertainties of the location and scale parameters of the population depend on the number of observation $n$ as a function of $1 / \sqrt{n-3}$, i.e. can be determined when $n \geq 4$. When the number of observations is small then the uncertainty value calculated by the usual method may differ significantly from the correct value. The given numerical example confirms this statement.
\end{abstract}

\section{Keywords:}

Measurement, Observation, Estimator, Uncertainty, Probability distribution.

\section{Introduction}

The evaluation of the uncertainty of the obtained results is an obligatory element of the processing of measurement observations. Guide [1] directly states: "When reporting the result of a measurement of a physical quantity, some quantitative indication of the quality of the result must be given so that those who use it can assess its reliability". In Guide [1] and the VIM [2] we can find the definition: ,uncertainty - parameter, associated with the result of a measurement, that characterizes the dispersion of the values that could reasonably be attributed to the measurand".

Here it should be noted that in this definition the uncertainty refers to the possible values of the measurand but not to an estimate of the measurand. This is important for the correct evaluation of the uncertainty of measurement in the development of multiple observations. The problem of evaluating the standard uncertainty of such measurements is not quite correctly presented even in the Guide [1] and other documents related to Guide.

From the very beginning of the implementation of Guide [1], some metrologists [3 - 9] pointed out that the estimation of Type A uncertainty is inconsistent with the definition of the "uncertainty".

Namely, using $n$ independent observations $x_{1}, x_{2}, \ldots x_{n}$, derived from a Gaussian (normal) population, which probability density distribution function (PDF) is

$$
p_{N p}(x \mid \mu, \sigma)=\frac{1}{\sqrt{2 \pi} \sigma} e^{-\frac{1}{2}\left(\frac{x-\mu}{\sigma}\right)^{2}},
$$

where " $\mu$ " is the unknown expected value and " $\sigma$ " is the unknown standard deviation, when measurand is $\mu$ the best estimator of $\mu$ is the arithmetic mean [1]:

$$
\bar{x}=\frac{1}{n} \sum_{i=1}^{n} x_{i} .
$$

This is explained [1] as: "In most cases, the best available estimate of the expectation or expected value $\mu_{q}$ of a quantity $q$ $\ldots$ is the arithmetic mean or average $q$ of the $n$ observations". In this article, we used " $x$ " instead of quantity $q$.

In [1] the Type A uncertainty is determined by the experimental variance $S^{2}$ of the observations, which estimates the variance $\sigma^{2}$ :

$$
S^{2}=\frac{1}{n-1} \sum_{i=1}^{n}\left(x_{i}-\bar{x}\right)^{2}
$$

Then the standard uncertainty (Type A) is defined as:

$$
u_{A}(x)=S(\bar{x})=\sqrt{\frac{S^{2}}{n}}=\frac{S}{\sqrt{n}} .
$$

However, the evaluation of uncertainty according to (4) has not exact theoretical justification. 
In the theory of estimation [10 - 13] when we estimate location parameter $\mu$ and scale parameter $\sigma$ of (1) (and also another population) the estimators $m$ for $\mu$ and $s$ for $\sigma$ are the random quantities. For these estimators, the corresponding probability density functions $p_{m}(m \mid \mu, \sigma), p_{s}(s \mid \sigma)$ are determined using the appropriate estimation method, for example, the Likelihood method (MLE) [10 - 13]. When the population is Gaussian, then the PDF of these estimates $\left(\bar{x}, s=S \sqrt{\frac{n}{n-1}}\right)$ are given as $[10]$ :

$$
p_{x}(\bar{x} \mid \mu, \sigma)=\frac{\sqrt{n}}{\sqrt{2 \pi} \sigma} e^{-\frac{n \cdot(\bar{x}-\mu)^{2}}{2 \sigma^{2}}}, p_{s}(s \mid \sigma)=\frac{2 \cdot\left(\frac{n}{2}\right)^{\frac{n-1}{2}}}{\sigma \cdot \Gamma\left(\frac{n-1}{2}\right)}\left(\frac{s}{\sigma}\right)^{n-2} e^{-\frac{n \cdot s^{2}}{2 \sigma^{2}}}, s>0 .
$$

From (5) the expected values and variances of mean $\bar{x}$ and experimental standard deviation $s=S \sqrt{\frac{n}{n-1}}$ are equal:

$$
\begin{gathered}
E(\bar{x} \mid \mu)=\mu, \operatorname{var}(\bar{x} \mid \sigma)=\frac{\sigma^{2}}{n}, \\
E(s \mid \sigma)=\sigma \sqrt{\frac{2}{n}} \frac{\Gamma\left(\frac{n}{2}\right)}{\Gamma\left(\frac{n-1}{2}\right)}, \operatorname{var}(s \mid \sigma)=\sigma^{2} \frac{n-1}{n}\left(1-\frac{2}{n-1}\left(\frac{\Gamma\left(\frac{n}{2}\right)}{\Gamma\left(\frac{n-1}{2}\right)}\right)^{2}\right) .
\end{gathered}
$$

Analogically from (6) and (7), the standard deviations of mean $\bar{x}$ and experimental standard deviation s described by wellknown formulas:

$$
\sigma_{\bar{x}}=\frac{\sigma}{\sqrt{n}}, \quad \sigma_{s}=\sigma \sqrt{\frac{n-1}{n}} \sqrt{\left(1-\frac{2}{n-1}\left(\frac{\Gamma\left(\frac{n}{2}\right)}{\Gamma\left(\frac{n-1}{2}\right)}\right)^{2}\right)} .
$$

From the first part of (8), we can see that "standard uncertainty" (4) of the mean value $\bar{x}$ is obtained by substitution of the population standard deviation $\sigma$ in standard deviation $\sigma_{\bar{x}}$ (8) into experimental standard deviation $\mathrm{S}$ in (4).

From the definition given in Guide [1] standard uncertainty has to be applied to the measurand, i.e. unknown expectation $\mu$ but not to the estimator, i.e. arithmetic mean $\bar{x}$, which value after measurement experiment is known, or is not random.

The same problem relates to evaluation uncertainty in other measurement procedures, for example, in linear regression, which is not analyzed in this article.

\section{The Aim of the Research}

The aim presented in the article is to explain the causes of the situation of incorrect determination of Type A standard uncertainty and to present a correct solution to this problem.

\section{Statistical Problems in Evaluation of Standard Uncertainty by Type A Method}

The basic problem in the evaluation of standard uncertainty by the Type A method is the theoretically unjustified transfer of statistical properties of estimators onto measurand properties [14].

As it was mentioned above, the calculation of the standard uncertainty according to (3), (4) ((5) in [1] chapter 4.2.3) does not correspond to the definition of uncertainty given in Guide [1] because relate to the estimator (arithmetic mean) but not an expectation - measurand. From the definition of uncertainty, it means, that "the dispersion of the values" is not applied to the estimate of the result but applied to the dispersion of the values of measurand (here expectation) around estimated result (here arithmetic mean [14]. The parameters of the dispersion of the estimators can be derived from the respective probability distribution functions $p_{m}(m \mid \mu, \sigma), p_{s}(s \mid \sigma)$. Here $m$ is an estimator of a location parameter $\mu$ of the population (not always expected as in Gaussian distribution) and $s$ is an estimator of a scale parameter $\sigma$ of the population 
(not always standard deviation as in Gaussian distribution). However, we must remember that in the theory of estimation location parameter $\mu$ and scale parameter $\sigma$ of the population $p_{p}(x \mid \mu, \sigma)$ are constant but unknown, and estimated values $m$ and $s$ are the random quantities.

Therefore, the standard deviations determined from distributions $p_{m}(m \mid \mu, \sigma), p_{s}(s \mid \sigma)$ relate to the possible dispersion of the estimator values around the relevant population parameters if the subsequent series of observations are registered. The standard deviation (and other all parameters) of estimators unequivocally depend on population parameters. Therefore in general determined standard deviation of the estimator can not be used directly to determine the uncertainty of the population parameter.

Another problem is that presented in Guide [1] the Type A uncertainty analysis practically only applies to the expectation of the population when the estimator - measurement result is an arithmetic mean. Theoretically, this applies only to Gaussian distribution. When the distribution of a population essentially differs from Gaussian then the arithmetic means may not be the "best" estimator of such population location parameter and standard deviation also may not be the adequate base to determinate standard uncertainty of measurand [14]. The Guide [1] and the Dictionary [2] clearly state that Type A evaluation of uncertainty method base on "statistical analysis of series of observations", however, apart from the method based on the experimental standard deviation of the random sample, no other methods are presented. For such populations, we cannot use a direct procedure that is related to the Gaussian population.

\section{Evaluation of Standard Uncertainty of Measurand by Solution of Inverse Problem}

It is clear that to determine the standard uncertainty of measurand $u_{A}(\mu)$ (and similarly the standard uncertainty $u_{A}(\sigma)$ when $\sigma$ is measurand) we must have probability density functions $p_{\mu}(\mu \mid m, s)$ (and also $p_{\sigma}(\sigma \mid s)$ ) of the population parameters, which for the given population depend on the determined from experiment the estimator values $\mathrm{m}$ and $\mathrm{s}$. After the measurement experiment the estimated numerical values $m$ and $s$ are known i.e. are not random, however, the possible values of unknown parameters $\mu$ and $\sigma$ of a population are random. The randomness of the parameters $\mu$ and $\sigma$ is understood as their possible values, for which in measurement experiments after processing of the observations could give the observed numerical values $m$ and $s$ obtained in a given experiment.

The distributions $p_{\mu}(\mu \mid m, s), p_{\sigma}(\sigma \mid s)$ of the possible values of the population parameters $\mu$ and $\sigma$ in the given experiment cannot be obtained directly using the estimation procedure. For this purpose, other procedures should be used. One of such procedures applied to uncertainty evaluation is based on the Bayesian approach. Various aspects and problems related to the choice of Bayesian method to uncertainty evaluation by Type A method are presented in numerous literature [4-9, 12]. In this method, the prior distributions $p_{\mu, p}(\mu) p_{\sigma, p}(\sigma)$ which represent the states of knowledge of the population parameters should be used. In such tasks, Jeffrey's rule is used to construct these prior distributions. Namely, for $\mu$ and $\sigma^{2}$ these a priori distributions are given as $[15,16]$ :

$$
p(\mu) \propto 1,-\infty \leq \mu \leq \infty, p\left(\sigma^{2}\right) \propto \frac{1}{\sigma^{2}}, 0 \leq \sigma^{2} \leq \infty .
$$

Choosing the proper a priori distributions is a fundamental problem of the Bayesian method that gives rise to many discussions on this subject.

The general method of the determination of the posterior distributions of the population parameters method of Type A uncertainty evaluation, which does not need any a priori distribution, is based on the solution of the inverse problem of uncertainty proposed and analyzed in detail in [14]. In this method previously the joint distribution $p_{m, s}(m, s \mid \mu, \sigma)$ of both estimators $m$ and $s$ should be determined. The distribution $p_{m, s}(m, s \mid \mu, \sigma)$ is a result of the solution so-called forward problem using an appropriate estimation method applied to population distribution $p_{p}(x \mid \mu, \sigma)$. In the next stage, when values of estimators $\mathrm{m}$ and $\mathrm{s}$ are known, the a posteriori joint distribution $p_{\mu, \sigma}(\mu, \sigma \mid m, s)$ of the location $\mu$ and scale $\sigma$ parameters of the population is determined by transformation of the estimators' joint distribution $p_{m, s}(m, s \mid \mu, \sigma)$ by Jacobian which is a ratio of estimate $(s)$ to scale parameter $(\sigma)$ of the population [14]:

$$
J(s, \sigma)=\frac{s}{\sigma}
$$

i.e. 


$$
p_{\mu, \sigma}(\mu, \sigma \mid m, s)=\frac{s}{\sigma} \cdot p_{m, s}(m, s \mid \mu, \sigma) .
$$

The distributions $p_{\mu}(\mu \mid m, s)$ of location $\mu$ and $p_{\sigma}(\sigma \mid s)$ of scale $\sigma$ parameters are determined by the integration of the joint distribution (11):

$$
\begin{gathered}
p_{\mu}(\mu \mid m, s)=\int_{0}^{\infty} \frac{s}{\sigma} \cdot p_{m, s}(m, s \mid \mu, \sigma) d \sigma, \\
p_{\sigma}(\sigma \mid s)=\int_{0}^{\infty} \frac{s}{\sigma} \cdot p_{m, s}(m, s \mid \mu, \sigma) d \mu=\left(\frac{s}{\sigma}\right) p_{s}(\sigma \mid s) .
\end{gathered}
$$

Type A standard uncertainties $u_{A}(\mu \mid s), u_{A}(\sigma \mid s)$ of the population parameters are determined after previous determination of the expected values $E(\mu \mid m, s), E(\sigma \mid s)$ and variances $\operatorname{var}(\mu \mid m, s), \operatorname{var}(\sigma \mid s)$ using PDF $p_{\mu}(\mu \mid m, s)$ and $p_{\sigma}(\sigma \mid s)$ by known formulas:

$$
\begin{aligned}
& E[\mu \mid m, s]=\int_{-\infty}^{\infty} \mu \cdot p_{\mu}(\mu \mid m, s) d \mu, E[\sigma \mid s]=\int_{-\infty}^{\infty} \sigma \cdot p_{\sigma}(\sigma \mid s) d \sigma, \\
& \operatorname{var}[\mu \mid s]=\int_{-\infty}^{\infty} \mu^{2} \cdot p_{\mu}(\mu \mid m, s) d \mu-E^{2}[\mu \mid m, s], \operatorname{var}[\sigma \mid s]=\int_{-\infty}^{\infty} \sigma^{2} \cdot p_{\sigma}(\sigma \mid s) d \sigma-E^{2}[\sigma \mid s], \\
& u_{A}(\mu \mid s)=\sqrt{\operatorname{var}[\mu \mid s],} u_{A}(\sigma \mid s)=\sqrt{\operatorname{var}[\sigma \mid s]} .
\end{aligned}
$$

For asymmetrical population distribution, $\frac{1}{\sigma} p_{p}\left(\frac{|x-\mu|}{\sigma}\right)$ the distribution $f_{\mu}(\mu \mid m, s)$ is symmetric also. Therefore the expected value $E(\mu \mid m, s)$ of $\mu$ always is equal to the estimated value $m$ :

$$
E(\mu \mid m, s)=E(\mu)=m
$$

For asymmetrical populations, the expected value $E(\mu \mid m, s)$ of a location parameter is not equal to the estimated value $m$ and is dependent on the estimated value $s$ of the scale parameter.

From (18) never expected value $E(\sigma \mid s)$ of scale parameter $\sigma$ never equals to estimated value $s$.

The properties of the expected values, variances, and standard uncertainties $u_{A}(\mu \mid s), u_{A}(\sigma \mid s)$ and also expanded uncertainties depend on the properties of PDF $p_{\mu}(\mu \mid m, s), p_{\sigma}(\sigma \mid s)$ and some observations. In [14] was shown, that independently of the kinds of population distribution, because with an unlimited increase of the ratio $|m-\mu| / s \rightarrow \infty$, and $\sigma / s \rightarrow \infty$ the distribution $f_{\mu}(\mu \mid m, s)$ decreases proportionally to the power $n$ of this ratio $(|m-\mu| / s)^{n}$ and the distribution $f_{\sigma}(\sigma \mid s)$ decreases proportionally to $(\sigma / s)^{n}$ :

$$
p_{\mu}(\mu \mid m, s) \underset{\frac{|\mu-m|}{s} \rightarrow \infty}{\longrightarrow} \sim \frac{1}{s \cdot\left(\frac{|\mu-m|}{s}\right)^{n}}, \quad p_{\sigma}(\sigma \mid s)_{\frac{\sigma}{s} \rightarrow \infty} \sim \frac{1}{s} \cdot\left(\frac{s}{\sigma}\right)^{n}
$$

Cumulative distributions, which is used for determination of expanded uncertainties $U_{p}(\mu \mid m, s)$ and $U_{p}(\sigma \mid s)$ ( $p$ is confidence level) due to (23):

$$
F_{\mu}(\mu \mid m, s)=\int_{-\infty}^{\mu} p_{\mu}(\mu \mid m, s) d \mu \sim \frac{1}{\left(\frac{|\mu-m|}{s}\right)^{n-1}}, F_{\sigma}(\sigma \mid s)=\int_{0}^{\sigma} p_{\sigma}(\sigma \mid s) d \sigma \sim\left(\frac{s}{\sigma}\right)^{n-1} .
$$

From (18) and (19) follows that independently on the population distribution: 
(i) the expanded uncertainties $U_{p}(\mu \mid m, s)$ and $U_{p}(\sigma \mid s)$ of population parameters can be determined when the number of observations $n \geq 2$;

(ii) the expected values $E(\mu \mid m, s)$ and $E(\sigma \mid s)$ (14) of population parameters can be determined when some observations $n \geq 3$;

(iii) independently on population distribution variances (15) of the location and scale parameters of the population have the multiplier $1 /(n-3)$, and as consequence, the standard uncertainties (16) of these parameters have the multiplier $1 / \sqrt{n-3}$ :

$$
u_{A}(\mu \mid s) \sim \frac{s}{\sqrt{n-3}} \quad, u_{A}(\sigma \mid s) \sim \frac{s}{\sqrt{n-3}} .
$$

Therefore standard uncertainties $u_{A}(\mu \mid s)=\sqrt{\operatorname{var}(\mu \mid s)}, u_{A}(\sigma \mid s)=\sqrt{\operatorname{var}(\sigma \mid s)}$ of the population parameters can be determined only if the number of observations is greater than 3, i.e.: $n \geq 4$.

For the Gaussian population the posterior distribution of location parameter $p_{\mu}(\mu \mid m, s)$ is given by [14]:

$$
p_{\mu}(\mu \mid m, s)=\frac{\Gamma\left(\frac{n}{2}\right)}{\sqrt{\pi} \cdot \Gamma\left(\frac{n-1}{2}\right) \cdot s} \cdot\left(1+\left(\frac{\mu-m}{s}\right)^{2}\right)^{-\frac{n}{2}} .
$$

Distribution (21) is well known as a Student type distribution with $v=n-1$ degrees of freedom [1], which is given not in standard form. From (21) the variance and standard uncertainty of $\mu$ are equal:

$$
\operatorname{var}(\mu \mid s)=\frac{s^{2}}{n-3}=\frac{S^{2}}{n} \cdot \frac{n-1}{n-3}, u_{A}(\mu \mid s)=\sqrt{\operatorname{var}(\mu \mid s)}=\frac{s}{\sqrt{n-3}}=\frac{s}{\sqrt{n}} \cdot \sqrt{\frac{n-1}{n-3}}, n \geq 4,
$$

These values are consistent with (20).

The posterior distribution $f_{\sigma}(\sigma \mid s)$ of scale parameter is given as.:

$$
f_{\sigma}(\sigma \mid s)=\frac{s}{\sigma} \cdot p_{s}(s \mid \sigma)=\frac{2 \cdot\left(\frac{n}{2}\right)^{\left(\frac{n-1}{2}\right)}}{s \cdot \Gamma\left(\frac{n-1}{2}\right)}\left(\frac{s}{\sigma}\right)^{n} e^{-\frac{n \cdot s^{2}}{2 \sigma^{2}}}, \sigma>0, s>0 .
$$

Distribution (23) is a well-known $\chi$ (Chi) - distribution with $v=n-1$ degrees of freedom [17]. When $\sigma$ is a measurand then from (23) the expected value, variance, and standard uncertainty are given as [14]:

$$
\begin{aligned}
& E(\sigma \mid s)=\sqrt{\frac{n}{2}} \cdot \frac{\Gamma\left(\frac{n}{2}-1\right)}{\Gamma\left(\frac{n-1}{2}\right)} \cdot s, n \geq 3 ; \operatorname{var}[\sigma \mid s]=\frac{n}{n-3} \cdot\left\{1-\frac{n-3}{2} \cdot\left[\frac{\Gamma\left(\frac{n}{2}-1\right)}{\Gamma\left(\frac{n-1}{2}\right)}\right]^{2}\right\} \cdot s^{2}, n \geq 4, \\
& u_{A}(\sigma \mid s)=\sqrt{\frac{n}{n-3}} \cdot \sqrt{1-\frac{n-3}{2} \cdot\left[\frac{\Gamma\left(\frac{n}{2}-1\right)}{\Gamma\left(\frac{n-1}{2}\right)}\right]^{2}} \cdot s=\sqrt{\frac{n-1}{n-3}} \cdot \sqrt{1-\frac{n-3}{2} \cdot\left[\frac{\Gamma\left(\frac{n}{2}-1\right)}{\Gamma\left(\frac{n-1}{2}\right)}\right]^{2}} \cdot S, n \geq 4
\end{aligned}
$$

After comparison uncertainties (4) and (18), (22), we can see that in Guide [1] uncertainty (4) relates to the arithmetic means $\left(u_{A}(\bar{x})\right.$ ) but uncertainty $(22) u_{A}(\mu)$ relates to the measurand $\mu$. From the analysis of (22), we can considerate that "uncertainty" $u_{A}(\bar{x})$ in (4) is the only approach of uncertainty $u_{A}(\mu)$ (22) and this approaching can be used only for a large number of observations:

$$
u_{A}(\mu) \approx \frac{s}{\sqrt{n}}, n \rightarrow \infty(n>>3)
$$


A similar consideration is applied to the standard uncertainty $u_{A}(\sigma)$ when the standard deviation $\sigma$ of the population is a measurand.

For the uniform population with distribution [13]:

$$
p_{U p}(x \mid \mu, \sigma)=\frac{1}{2 \sigma}\left\{\begin{array}{c}
1, \mu-\sigma \leq x \leq \mu+\sigma, \\
0, \quad \text { othevise }
\end{array}\right.
$$

where $\mu$ is a midrange and $\sigma$ is a half-range, a posteriori distributions $p_{\mu}(\mu \mid m, s)$ of $\mu$ and $p_{\sigma}(\sigma \mid s)$ of $\sigma$ are given by equations [14]:

$$
\begin{gathered}
p_{\mu}(\mu \mid m, s)=\frac{n-1}{2 s} \cdot \frac{1}{\left(1+\frac{|\mu-m|}{s}\right)^{n}} . \\
p_{\sigma}(\sigma \mid s)=\frac{n(n-1)}{s}\left(\frac{s}{\sigma}\right)^{n}\left(1-\frac{s}{\sigma}\right), \frac{s}{\sigma} \leq 1 .
\end{gathered}
$$

where estimators of population parameters are determined by a first (minimal) $x_{s 1}$ and a last (maximal) $x_{s n}$ observation after sorting [13]:

$$
m=\frac{x_{s n}+x_{s 1}}{2}, s=\frac{x_{s n}-x_{s 1}}{2}, S=s \cdot \frac{n+1}{n-1} .
$$

From (28) the variance and standard uncertainty of the midrange $\mu$ of the population are:

$$
\operatorname{var}(\mu \mid s)=\frac{2}{n-2} \cdot \frac{s^{2}}{n-3}, u_{A}(\mu \mid s)=\sqrt{\operatorname{var}(\mu \mid s)}=\sqrt{\frac{2}{n-2}} \cdot \frac{s}{\sqrt{n-3}}=\sqrt{\frac{2}{n-2}} \cdot \frac{n-1}{n+1} \cdot \frac{s}{\sqrt{n-3}}, n \geq 4
$$

From (29) the expectation, variation, and standard uncertainty of the half range $\sigma$ of population, when experimental value $s$ is determined, are:

$$
E(\sigma \mid s)=\frac{n}{n-2} \cdot s, n \geq 3, \operatorname{var}(\sigma \mid s)=\frac{2 n}{(n-2)^{2}} \cdot \frac{s^{2}}{n-3}, u_{A}(\sigma \mid s)=\sqrt{\operatorname{var}(\sigma \mid s)}=\frac{\sqrt{2 n}}{n-2} \cdot \frac{s}{\sqrt{n-3}} n \geq 4
$$

We can see that standard uncertainty in (31) and (32) of the parameters $\mu$ and $\sigma$ for the uniform distribution are also consistent with (20).

In [1-2] expanded uncertainty is defined as the product of the coverage factor $k_{p, \mu}$, and the standard uncertainty of measurement. For example, when measurand is $\mu$ then:

$$
U_{p}(\mu)=k_{\mu, p} \cdot u_{A}(\mu \mid s)
$$

Because the standard uncertainty $u_{A}(\mu \mid s)$ exists when $n \geq 4$ therefore the value of a coverage factor $k_{p, \mu}$ can also be determined only when $n \geq 4$ :

$$
k_{\mu, p}=\frac{U_{p}(\mu)}{u_{A}(\mu \mid s)} .
$$

For the Gaussian population from (23) $k_{p, \mu}$ is given by:

but not $t_{p}(n-1)$ as in [1].

$$
k_{p, \mu}=t_{p}(n-1) \cdot \sqrt{\frac{n-3}{n-1}},
$$

When the number of observations is $n=2$ and $n=3$ the standard uncertainties of population parameters cannot be determined and due to this coverage factors $k_{p, \mu}$ are not exist. This problem can be solved using suitable a priori distribution of $\mu$. For example, from a physical point of view, the value of $\mu$ cannot be less than $\mu_{L}$ and not more than $\mu_{U}$, i.e. in the simplest case

Therefore, the posterior distribution of $\mu$ is given as:

$$
p_{a, \mu}(\mu)=\frac{1}{\mu_{U}-\mu_{L}}\left\{\begin{array}{c}
1, \mu_{L} \leq \mu \leq \mu_{U} \\
0, \text { otherwise. }
\end{array}\right.
$$

$$
p_{p, \mu}(\mu \mid m, s)=\frac{p_{\mu}(\mu \mid m, s)}{F_{\mu}\left(\frac{\mu_{U}-m}{s}\right)-F_{\mu}\left(\frac{\mu_{L}-m}{s}\right)}\left\{\begin{array}{c}
1, \mu_{L} \leq \mu \leq \mu_{U}, \\
0, \text { otherwise. }
\end{array}\right.
$$


For the distribution (37) the expected value and variance of $\mu$ can be determined for any $n \geq 2$ :

$$
\begin{gathered}
E_{p}(\mu \mid m, s)=\frac{1}{F_{\mu}\left(\frac{\mu_{U}-m}{s}\right)-F_{\mu}\left(\frac{\mu_{L}-m}{s}\right)} \int_{\mu_{L}}^{\mu_{U}} \mu \cdot p_{\mu}(\mu \mid m, s) d \mu, \\
\operatorname{var}_{p}(\mu \mid m, s)=\frac{1}{F_{\mu}\left(\frac{\mu_{U}-m}{s}\right)-F_{\mu}\left(\frac{\mu_{L}-m}{s}\right)} \int_{\mu_{L}}^{\mu_{U}} \mu^{2} \cdot p_{\mu}(\mu \mid m, s) d \mu-E_{p}^{2}(\mu \mid m, s) .
\end{gathered}
$$

Finally, posterior standard uncertainty of $\mu$ is:

$$
u_{A, p}(\mu)=\sqrt{\operatorname{var}_{p}(\mu \mid m, s)}, n \geq 2 .
$$

Independently on the population, the same properties have coverage factors $k_{p L, \sigma}$ and $k_{p L, \sigma}$ which corresponded to lower $U_{p L}(\sigma)$ and the upper $U_{p U}(\sigma)$ limits (because distribution $p_{\sigma}(\sigma \mid s)$ always is asymmetrical) of the confidence interval:

$$
k_{p L, \sigma}=\frac{U_{p L}(\sigma)}{u_{A}(\sigma \mid s)}, k_{p U, \sigma}=\frac{U_{p U}(\sigma)}{u_{A}(\sigma \mid s)} .
$$

\section{Example of Type A Uncertainty Evaluation}

In Annex H.2 Simultaneous resistance and reactance measurement (GUM [1]) the resistance $R$, the reactance $X$, an impedance $Z$ of a circuit element are determined by measuring the amplitude $\mathrm{V}$ of a sinusoidally-alternating potential difference across its terminals, the amplitude I of the alternating current passing through it, and the phase-shift angle $\phi$ of the alternating potential difference relative to the alternating current.

The set of the $n=5$ results of measurement of the amplitude $\mathrm{V}(\mathrm{V})$ current I (mA) and phase-shift angle $\phi(\mathrm{rad})$ are included in Table H.2 [1]. The experimental standard deviation of means (which are used as standard uncertainties to determinate combined standard uncertainties) are calculated using (4) $s(V)=0.0032, s(I)=0.0095$, and $s(\phi)=0.00075$ are given in this Table H.2 [1].

If we determine standard uncertainties of voltage, current and phase-shift angle by (21) we obtain the following values: $u_{A}(V) \approx 0.00454, u_{A}(I) \approx 0.013, u_{A}(\phi) \approx 0.00116$, which are $\sqrt{(n-1) /(n-3)}=\sqrt{2}$ (about 41\%) bigger than presented in Table H.2 [1] values.

Therefore calculated and presented in Table H.3 [1] values of combined uncertainties (absolute and relative) of resistance $R: u_{\mathrm{c}}(R)=0,071 \Omega$, reactance $X: u_{\mathrm{c}}(X)=0,295 \Omega$ and impedance $Z: u_{\mathrm{c}}(Z)=0,236 \Omega$ are $\sqrt{2}$ as small as they should be!

We can see that for the small number of observations using (4) to determinate standard uncertainty of measurand (here $\mu$ ) causes significant inaccuracies. It should be noted, that Guide [1] also clearly indicated this inaccuracy. Namely in E4.3 is stated, "Evaluations based on repeated observations are not necessarily superior to those obtained by other means". And next in E4.4 [1] we can find that: "the relative standard deviation of $s(q)$, which is given by the ratio $\sigma[s(q)] \sigma(q)$ and which can be taken as a measure of the relative uncertainty of $s(q)$, is approximately $[2(n-1)]-1 / 2$. This "uncertainty of the uncertainty" of $q$, which arises from the purely statistical reason of limited sampling, can be surprisingly large; for $n=10$ observations it is 24 percent".

\section{Conclusions}

The following conclusions apply to all population models $p_{p}(x \mid \mu, \sigma)$, which are described by two parameters of location $\mu$ and scale $\sigma$ and for which a variance exists.

According to the definition, the uncertainty refers to measurand and not to the measurand estimator. Therefore, when the standard uncertainty of measurand is determined based on the $n$ independent random observations $x_{1}, x_{2}, \ldots x_{n}$, which are consistent with an assumed model $p_{p}(x \mid \mu, \sigma)$, we must use probability distributions of this measurand, but not probability distributions of the estimator of this measurand.

When measurand is a population location parameter $\mu$ then standard uncertainty $u_{A}(\mu)$ may only be determined from density distribution $p_{\mu}(\mu \mid m, s)$ (where $m$ and $s$ are the estimators of population parameters $\mu$ and $\sigma$ ), but not from density 
distribution $p_{m}(m \mid \mu, \sigma)$ of estimator $m$. We similarly proceed when measurand is a population scale parameter $\sigma$, i.e. by using density distribution $p_{\sigma}(\sigma \mid s)$.

The density distributions $p_{\mu}(\mu \mid m, s)$ and $p_{\sigma}(\sigma \mid s)$ can be determined from the joint distribution $p_{\mu, \sigma}(\mu, \sigma \mid m, s)$ which in turn is determined by transformation joint distribution $p_{m, s}(m, s \mid \mu, \sigma)$ of estimators by Jacobian which is a ratio of estimate $(s)$ to scale parameter $(\sigma)$ of population.

The standard uncertainty determined from the estimator distribution (when substituting $\sigma$ for its estimator $S$ ) is $\sqrt{(n-1) /(n-3)}$ times less than the correct value of the uncertainty determined from the distribution of the population parameter. Independently of the population distribution the correct standard uncertainty of measurand can be determined when some observations are more than 3 , i.e. $n \geq 4$. Therefore, if the number of observations is small the evaluation of standard uncertainty according to the method presented in the Guide significantly underestimates its correct value. The method of Guide can only be used for large $n$.

When $n=2$ and 3 the standard uncertainty of measurand can be determined only by using a priori density distribution of measurand.

Since the correct value of the standard uncertainty differs from the GUM value, to present the expanded uncertainty as to the product of the standard uncertainty and the coverage factor the value of this factor must be recalculated.

\section{Acknowledgments}

The authors express their gratitude to the staff of the Department of Information-Measuring Technologies of Lviv Polytechnic National University, Ukraine, for the assistance in the preparation of this article.

\section{Conflict of Interests}

Conflict of interest while writing, preparing, and publishing the article as well as mutual claims by the co-authors is absent.

\section{References}

[1] JCGM 100:2008 Evaluation of measurement data-Guide to the expression of uncertainty in measurement Joint Committee for Guides in Metrology, 2008.

[2] JCGM 200:2012 International vocabulary of metrology - Basic and general concepts and associated terms (VIM) 3rd edition, 2012.

[3] W. Bich, M. Cox, C. Michotte, "Towards a new GUM — an update, Metrologia, no.53, 2016.

[4] R. Kacker, A. Jones, "On use of Bayesian statistics to make the Guide to the Expression of Uncertainty in Measurement consistent", Metrologia, no.40, pp. 235-248, 2003.

[5] M. Cox, K. Shirono, "Informative Bayesian Type A uncertainty evaluation, especially applicable to a small number of observations", Metrologia, no.54, pp.642-652, 2017.

[6] K. Weise, W. Wöger, “A Bayesian theory of measurement uncertainty”, Meas. Sci. Technol., no.3, pp.1-11, 1992.

[7] J. Berger, Statistical Decision Theory and Bayesian Analysis. New York: Springer, 1985.

[8] I. Lira, W. Wöger, "Bayesian evaluation of the standard uncertainty and coverage probability in a simple measurement model", Meas. Sci. Technol., no.12, pp.1172-9, 2001.

[9] I. Lira, W. Wöger, "Evaluation of Repeated Measurement from the Viewpoint of Conventional and Bayesian Statistics. Advanced Mathematical and Computational Tools in Metrology VII", World Scientific Publishing Co., pp.73-84, 2006.

[10] M. Fisz, Probability Theory and Mathematical Statistics. John Wiley \& Sons, 1967.

[11] Handbook of Applicable Mathematics. Vol.VI: Statistics, part A, John Wiley and Sons, 1984.

[12] E. Lehmann, Theory of point estimation, Wiley, 1983.

[13] H. Cramer, Mathematical methods of statistics. Princeton University Press.

[14] M. Dorozhovets, "Forward and inverse problems of Type A uncertainty evaluation", Measurement, vol.165, 2020. https://doi.org/10.1016/j.measurement.2020.108072

[15] H. Jeffreys, Theory of probability, 3-d edition, Oxford University Press (Oxford), 1983.

[16] P. Christian, N. Chopin, J. Rousseau, H. Jeffreys's, “Theory of Probability Revisited. Statistical Science, Institute of Mathematical Statistics", vol.24, no.2, pp.141-172, 2009.

[17] M. Evans, N. Hastings, B. Peacock, Chi Distribution in Statistical Distributions, New York, Wiley, 2000. 